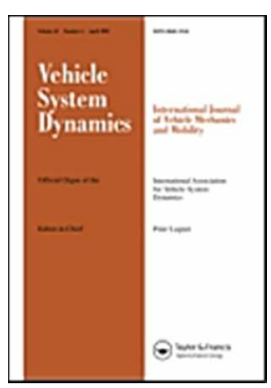

\title{
On the Coherency of Dynamic Load Estimates for Vehicles on Flexible Structures
}

\begin{tabular}{|r|l|}
\hline Journal: & Vehicle System Dynamics \\
\hline Manuscript ID: & Draft \\
\hline Manuscript Type: & Special Issue Paper \\
\hline Date Submitted by the Author: & n/a \\
\hline Keyworde List of Authors: & $\begin{array}{l}\text { Mitra, Mainak; University of Michigan,Ann Arbor, Mechanical Engineering } \\
\text { Gordon, Tim; University of Michigan, UMTRI; }\end{array}$ \\
\hline & $\begin{array}{l}\text { Vehicle-bridge interaction, Vehicle-Infrastructure interaction, Modal } \\
\text { duty vehicles, Tyre dynamics }\end{array}$ \\
\hline
\end{tabular}

SCHOLARONE $^{\text {'x }}$

Manuscripts 


\title{
On the Coherency of Dynamic Load Estimates for Vehicles on Flexible Structures
}

\author{
Mainak Mitra ${ }^{1}$ \\ Graduate Student \\ Department of Mechanical Engineering, University of Michigan \\ G. G. Brown Laboratory, 2350 Hayward \\ Ann Arbor, Michigan 48109, USA \\ Timothy Gordon ${ }^{2}$ \\ Professor \\ University of Michigan Transportation Research Institute (UMTRI) \\ 2901 Baxter Road \\ Ann Arbor, Michigan 48109, USA
}

Correspondence details:

Address: 2200, EECS Building, 1301 Beal Avenue, Ann Arbor, MI, USA 48109-2122

Tel: +1-734-276-1647; e-mail: mitram@umich.edu

\begin{abstract}
This work was supported by the U.S. Department of Commerce, National Institute of Standards and Technology (NIST) Technology Innovation Program (TIP) under Cooperative Agreement Number 70NANB9H9008.
\end{abstract}

\footnotetext{
${ }^{1}$ Corresponding author

${ }^{2}$ Current affiliation: University of Lincoln, School of Engineering, Brayford Pool, Lincoln LN7TS,United Kingdom 


\section{On the Coherency of Dynamic Load Estimates for Vehicles on Flexible Structures}

This paper develops a novel form of a well-known signal processing technique, so as to be applicable to the interaction between a heavy truck and a supporting bridge structure. Motivated by the problem of structural health monitoring of bridges, a new modal coherency function is defined. This relates the input action of moving wheel loads to the dynamic response of the bridge, including the effects of unevenness of the road surface and the vertical dynamics of the truck suspension. The analysis here is specifically aimed at future experimental testing - the validation of axle load estimators obtained from sensors on the truck. It is applicable even when no independent 'ground truth' for the dynamic loads is available. The approach can be more widely used in the analysis of dynamic interactions involving suspended moving loads on deformable structures, e.g. for structural vibrations due to high-speed trains.

Keywords: vehicle-bridge interaction; vehicle-infrastructure interaction; modal analysis; algorithms; truck; tyre dynamics

\section{INTRODUCTION}

In this paper we consider the dynamics of a heavy truck moving over a bridge structure. Although this is a much studied problem [1-4], there are gaps in the literature from the perspective of signal processing; specifically there is currently no practical technique to test whether a measured structural response is adequately explained by the force time-histories of an individual vehicle estimated for multiple axle loads [4]. This is important for structural health monitoring which is based on a presumed knowledge of the input forces [1,2]. Assuming the bridge responds linearly to the applied loads, this may be addressed by evaluating the coherency of the input and output signals; but, given the continuous nature of the bridge structure, plus excitations arising from moving dynamic loads, it is not obvious how suitable coherency measures should be defined. There are other challenges implicit in the problem, especially when the duration of forcing input 
is limited, as is in the case of a truck moving at highway speeds over a relatively short bridge structure.

For a truck moving over a bridge with an uneven road surface, the vehicle-structure interaction involves the dynamic wheel loads causing bridge to deflect vertically, which in turn influences the dynamic inputs to the vehicle suspension. Note that the dynamic loads at the interface may differ considerably from the static loads exerted by the vehicle when it is at rest or in the absence of road unevenness[2]. It is therefore of considerable interest for the purposes of design, analysis and monitoring of bridge structures to have a sufficiently accurate estimate of the dynamic forces $[1,3,4]$. However, accurate direct measurement of such forces using sensors mounted close to the vehicle-structure interface such as on the tyre is inherently difficult and currently infeasible for widespread use $[5,6]$. Instead, indirect methods for estimation of the vehicle loads have been established [7-12]. These involve vehicle-mounted sensors such as accelerometers which, together with simple dynamic models and signal processing, provide indirect estimates of the dynamic loads.

In simulation it is possible to evaluate estimator performance by comparing the 'actual' forces exerted at the interface, but in physical experiments no such reference exists. To address this, the aim is to analyze the problem from both sides of the vehicle-structure interface. We note that previous efforts have been made to estimate dynamic loading purely based on the structural response [13-15]. However, such methods are unduly dependent on knowing an accurate model of the structure, and do not offer any independent measure of validation. This paper develops and validates the coherency analysis using high-fidelity simulations of the vehicle-structure interaction [16-17] and tests the coherency performance of a number of truck-based load estimators, including Kalman filters [18]. 
There are two critical challenges in the determination of input-output coherency in this situation. First, the vertical tyre loads are moving over a continuous structure; from the perspective of the structure the dynamic input is a function of both position and time, i.e. the problem is strongly multi-input-multi-output. Secondly, for the bridge structure considered, the truck is only in contact with the flexible bridge structure for a few seconds. Even for a fixed load, such a small data set proves to be insufficient for conventional coherency analysis based on Fourier transforms and cross-spectral density estimates. Also, considering the low structural damping of the bridge, the method fails to provide any reliable or repeatable measure of inputoutput coherency.

In the new analysis, assumed modes are used, derived from the eigenvectors of a linearized bridge model. The complex eigenvalues are then estimated by a form of system identification, resolving estimated forces and structural responses according to the assumed eigenvectors and performing parameter estimation from the input-output data. A frequencyindependent modal coherency measure is defined which has properties similar to that of the classical coherency measure. Further, a measure of pooled coherency is defined, based on the individual modal coherencies. This measure indicates the coherency across a number of modes of the structure, each corresponding to a separate natural frequency. It is shown that under certain idealized conditions the modal estimates will match the frequency-averaged classical coherency. The performance of the defined measures is extensively tested in simulation by introducing corrupting noise for both vehicle loads and bridge responses. Although real vehicle sensor values are available from field testing, corresponding bridge response data are as yet unavailable. While further field testing needs to be carried out to obtain this data, the available data are used in conjunction with a Kalman filter to obtain realistic force estimates. Both of the coherency measures are further tested by combining the experimentally determined forces and simulated 
bridge responses, hence removing any dependency on truck model fidelity. Different levels of random output noise are artificially added to study the effect on estimation trends.

The focus of this paper is on method development, and further experimental data are needed to validate the functionality of the approach across the range of existing physical bridge structures. It may be that the same approach will be applicable to the dynamics of trains on flexibly supported tracks [19-20]. Here we will limit analysis to a finite element model of a particular bridge structure where modal properties are known.

\section{MOTIVATION}

\subsection{Classical measure of coherency}

Let $u$ be the true input signal to a linear time invariant system corrupted by noise $m$ and let $v$ be the true output corrupted by noise $n$. Let $x$ be the estimated input signal and $y$ the measured output signal.

$$
x(t)=u(t)+m(t) ; y(t)=v(t)+n(t)
$$

Assuming that the noise signals are uncorrelated with the signals, the squared coherency between the signals $x$ and $y$ is defined as [21]:

$$
\gamma_{x y}^{2}(f):=\frac{\left|G_{x y}(f)\right|^{2}}{G_{x x}(f) G_{y y}(f)}=\frac{1}{1+\alpha(f)+\beta(f)+\alpha(f) \beta(f)}
$$

where $G_{x y}(f)$ is the cross spectral density between $x$ and $y ; G_{x x}$ and $G_{y y}$ are the autospectral density of $x$ and $y$ respectively. Further, $\alpha(f)$ and $\beta(f)$ are the input and output noise to signal ratios (NSR) defined as the following:

$$
\alpha(f)=\frac{G_{m m}(f)}{G_{u u}(f)} \quad ; \quad \beta(f)=\frac{G_{n n}(f)}{G_{v v}(f)}
$$




\subsection{Limitations of FFT based estimation}

It may be seen that the classical coherence measure $\gamma_{x y}^{2}(f)$ is an indicator of how precisely the measured outputs are explained by the estimated inputs applied to a linear system. In case of a perfectly linear system, with no input and output noise, $(\alpha(f)=\beta(f)=0)$ this value equals 1; it drops towards 0 as the noise to signal ratios increase, and is also reduced in the presence of nonlinearities.

In practice, an estimate of this measure, $\hat{\gamma}_{x y}^{2}(f)$, is obtained by estimating the power spectra of the signals by using Fast Fourier Transform (FFT) based techniques applied to weighted windows of the time-domain data of the signals $x$ and $y$. The details of the procedure may be found in [22]. The values of $\hat{\gamma}_{x y}^{2}$ obtained give an accurate estimate of the true value $\gamma_{x y}^{2}$ if the time-windows considered for averaging are significantly longer than the settling time of the linear system from which the signals are obtained. If that is not the case, the effects of the initial conditions inherited from a previous window will affect the subsequent one, degrading the accuracy of $\hat{\gamma}_{x y}^{2}$. This effect is illustrated in Figure 1.

In Figure $1, \hat{\gamma}_{x y}^{2}$ is obtained from the inputs and outputs of a $2^{\text {nd }}$ order linear system with natural frequency $10 \mathrm{~Hz}$, with no noise added to corrupt the signals. The signals are each $5 \mathrm{~s}$ long and sampled at $200 \mathrm{~Hz}$. A Hamming window of $0.5 \mathrm{~s}$ is used. Here, $\gamma_{x y}^{2}=1$ so the estimator should approximate this across all frequencies. It is seen that in Figure 1(a), when the damping of the system is relatively high and the settling time low compared to the window length, the estimation process performs well. However, the performance is degraded in Figure 1(b) when the damping is reduced and the settling time increases to beyond the window length. 


\section{METHODS}

\subsection{Proposed modal squared coherency}

The limitations of $\hat{\gamma}_{x y}^{2}$ make it inapplicable to the dynamic interaction of a vehicle on a short bridge, which possesses several low-frequency modes which are lightly damped. It might be that a longer time window, or synthesis of data from multiple runs could be used to compensate. However, such tests are unlikely to be feasible, and will probably require closing the structure to all traffic but the test vehicle. Thus, to retain the useful properties of the squared coherency function while taking account of the special circumstances mentioned, we propose a new coherency measure and a method of estimating it. In the context of vehicle-structure interaction, we assume a linear structure and a corresponding set of modes with known mode-shapes. Each mode has a modal force as input and a modal response as output which might be obtained through resolution of estimated tyre loads and measured bridge response using knowledge of mode-shapes by techniques described later.

The modal squared coherency measure is to be based on time responses resolved into a set of assumed modes of the bridge structure; it is defined for mode $i$ as:

$$
\gamma_{m, i}{ }^{2}:=\frac{\left|\mathbb{E}\left[y_{i}(t) \hat{y}_{i}(t)\right]\right|^{2}}{\left(\mathbb{E}\left[y_{i}(t)^{2}\right]\right)\left(\mathbb{E}\left[\hat{y}_{i}(t)^{2}\right]\right)}
$$

where $y_{i}$ represents the actual response (displacement, velocity or acceleration) in mode $i$ and $\hat{y}_{i}$ is the corresponding estimated output based on the resolved forcing input $x$. In this, $\hat{y}_{i}$ is obtained by system identification of the modal transfer function $\widehat{H}_{i}$, representing a second-order LTI system - see Figure 2. Parameter identification follows by choosing the estimated modal damping ratio and natural frequency to minimize the RMS error between the measured and estimated modal responses, i.e. 


$$
\hat{\xi}_{i}, \widehat{\omega}_{n, i}=\arg \min \left\{f\left(\hat{\xi}_{i}, \widehat{\omega}_{n, i}\right)=\sqrt{\left(\sum_{t=0}^{t_{f}}\left(y_{i}(t)-\hat{y}_{i}(t)\right)^{2}\right) / N_{t}}\right\}
$$

where $\hat{y}_{i}=\widehat{H}_{i}\left(\hat{\xi}_{i}, \widehat{\omega}_{n, i}\right)\{x\}, t_{f}$ is the final time step of available data and $N_{t}$ is the number of time steps from 0 to $t_{f}$.

As a consequence of the Cauchy-Schwarz inequality [23] the value of $\gamma_{m, i}{ }^{2}$ is limited between 0 and 1 . According to the definition, it equals 1 when there is a perfect match between predicted and measured response in the mode, and drops to 0 when the inputs and outputs are completely uncorrelated. For any two signals $x$ and $y$ their cross-correlation and spectral density functions are related by [21]:

$$
\mathbb{E}[x(t) y(t+\tau)]=\int_{0}^{\infty}\left(R e\left[G_{x y}(f)\right] \cos 2 \pi f \tau-\operatorname{Im}\left[G_{x y}(f)\right] \sin 2 \pi f \tau\right) d f
$$

For $\tau=0$ this reduces to

$$
\mathbb{E}[x(t) y(t)]=\int_{0}^{\infty} \operatorname{Re}\left[G_{x y}(f)\right] d f
$$

Assuming (i) uncorrelated noise and signals, (ii) wide-sense stationarity (i.e. the true mean and co-variance of the signals do not change with respect to time) and (iii) $H_{i}(f)$ to be a linear system (of course this is implicit in the transfer function model), we have

$$
G_{y_{i} y_{i}}(f)=\left|H_{i}(f)\right|^{2} G_{u u}(f)+G_{n_{i} n_{i}}(f)
$$

Also,

$$
G_{n_{i} n_{i}}(f)=\frac{\beta_{i i}(f)}{1+\beta_{i i}(f)} G_{y_{i} y_{i}}(f) \quad \text { and } \quad G_{u u}(f)=\frac{1}{1+\alpha(f)} G_{x x}(f)
$$

Thus,

$$
G_{y_{i} y_{i}}(f)=\frac{1+\beta_{i i}(f)}{1+\alpha(f)}\left|H_{i}(f)\right|^{2} G_{x x}(f)
$$


Similarly,

$$
G_{y_{i} \hat{y}_{i}}(f)=\frac{1}{1+\alpha(f)} H_{i}(f) \widehat{H}_{i}^{*}(f) G_{x x}(f) \quad \text { and } \quad G_{\hat{y}_{i} \hat{y}_{i}}(f)=\left|\widehat{H}_{i}(f)\right|^{2} G_{x x}(f)
$$

It follows from (4)-(9):

$$
\gamma_{m, i}^{2}:=\frac{\left|\mathbb{E}\left[y_{i} \hat{y}_{i}\right]\right|^{2}}{\left(\mathbb{E}\left[y_{i}{ }^{2}\right]\right)\left(\mathbb{E}\left[\hat{y}_{i}{ }^{2}\right]\right)}=\frac{\left|\int_{0}^{\infty} \operatorname{Re}\left[G_{y_{i} \hat{y}_{i}}(f)\right] d f\right|^{2}}{\left(\int_{0}^{\infty} \operatorname{Re}\left[G_{y_{i} y_{i}}(f)\right] d f\right)\left(\int_{0}^{\infty} \operatorname{Re}\left[G_{\hat{y}_{i} \hat{y}_{i}}(f)\right] d f\right)}
$$

Hence,

$$
\gamma_{m, i}^{2}=\frac{\left|\int_{0}^{\infty} \frac{1}{1+\alpha(f)} \operatorname{Re}\left[H_{i}(f) \widehat{H}_{i}^{*}(f) G_{x x}(f)\right] d f\right|^{2}}{\left(\int_{0}^{\infty} \frac{1+\beta_{i i}(f)}{1+\alpha(f)} \operatorname{Re}\left[\left|H_{i}(f)\right|^{2} G_{x x}(f)\right] d f\right)\left(\int_{0}^{\infty} \operatorname{Re}\left[\left|\widehat{H}_{i}(f)\right|^{2} G_{x x}(f)\right] d f\right)}
$$

It is apparent from equation (13) that in the presence of input or output errors $\alpha(f) \neq 0$ or $\beta_{i i}(f) \neq 0, \gamma_{m, i}{ }^{2}$ will decrease its value to below 1 . Also, in the case of non-linearities in the original model, estimation of the linear model will not be perfect and the estimated response will not fit the measured response leading to a drop in coherency. Thus, the proposed modal squared coherency behaves in the same manner as the classical measure, possessing the very properties that were attractive for its application to the problem.

In fact, under the assumption that (i) $\alpha(f)=\alpha$ and $\beta_{i i}(f)=\beta$ are frequency-independent constants and (ii) estimation is perfect, $H_{i}(f)=\widehat{H}_{i}(f)=H(f)$, equation (13) reduces to:

$$
\gamma_{m, i}{ }^{2}=\frac{\left|\int_{0}^{\infty} \frac{1}{1+\alpha} \operatorname{Re}\left[|H(f)|^{2} G_{x x}(f)\right] d f\right|^{2}}{\left(\int_{0}^{\infty} \frac{1+\beta_{i i}}{1+\alpha} \operatorname{Re}\left[|H(f)|^{2} G_{x x}(f)\right] d f\right)\left(\int_{0}^{\infty} \operatorname{Re}\left[|H(f)|^{2} G_{x x}(f)\right] d f\right)}=\frac{1}{1+\alpha+\beta_{i i}+\alpha \beta_{i i}}=\gamma_{x y_{i}}^{2}
$$

Hence in this special case, $\gamma_{m, i}{ }^{2}$ matches the classical definition $\gamma_{x y_{i}}^{2}$ in equation (2). And while $\gamma_{m, i}{ }^{2}$ requires additional information (i.e. mode-shape data), it does not suffer from the drawbacks of $\hat{\gamma}_{x y}^{2}$ estimation. The system identification step allows us to work with a limited set of data points, and there is only one frequency independent value of squared coherency obtained for each mode, clearly indicating how well the estimated inputs explain the measured outputs at 
the modal frequency. It must be noted that a low value of coherency might result from a structure wherein non-linearities dominate or whose modes are not known very well.

We now test the performance of $\gamma_{m, i}{ }^{2}$ for a single mode in a case where the input and output signals are suitably sparse. Figure 3 shows the variation in modal coherency for a mode with a natural frequency of $1.59 \mathrm{~Hz}$, damping ratio of 0.05 and the consequent settling time of $8 \mathrm{~s}$. The signals used for estimation are $5 \mathrm{~s}$ in length, sampled at $40 \mathrm{~Hz}$, so the signals are sparser than those used in the FFT based estimate of Figure 1. Here $\gamma_{m, i}{ }^{2}$ is reliably estimated to be 1 when noise is absent, and decreases monotonically with increasing noise to signal ratios as expected. It is also seen that the measure is more sensitive to input noise than to output noise, which is to be expected, due to the nature of system identification wherein input noise corrupts both the input and subsequently the estimated output, unlike output noise which corrupts only the measured output.

\subsection{Optimization Routine}

An objective function surface for a typical mode is shown in Figure 4(a). The surface is given by the function $f\left(\hat{\xi}_{i}, \widehat{\omega}_{n, i}\right)$ of equation (5). The red dot indicates the desired global minimum where $y_{i}(t)$ is identically equal to $\hat{y}_{i}(t)$ and therefore $\widehat{H}_{i}$ equals $H_{i}$ exactly if there is no noise. It may be seen that the function is not globally convex, but it is locally so. Although, the detailed analysis of the initial conditions and parameters required for convergence of a routine for a general case are beyond the scope of this study, it may be reasoned that due to the nature of response of a second-order LTI system, the value of the objective function will increase only if the input has significant frequency content near $\widehat{\omega}_{n, i}$, when $\widehat{\omega}_{n, i}$ is far away from $\omega_{n, i}$, which translates to convexity near the minima. Figure 4(b) shows that addition of random input and output time 
domain noise signals only increases the error in the frequency domain and changes the minimum value of the objective function but does not affect the minima as the function retains its shape.

It must be noted, that noise signals which are deterministic and arise due to errors in mode-shapes will affect the objective function differently than random signals. Thus, supplying highly inaccurate initial conditions or parameters to the system identification routine might also cause an artificial drop in the estimate due to non-convergence in certain modes. For the simulated results in the study, the initial values of $\widehat{\omega}_{n, i}$ were selected in randomly from a region around $\omega_{n, i}$ with a standard deviation of $5 \%$ of the value of $\omega_{n, i}$.

It may also be seen that the objective function does not have a strong dependence on the damping parameter $\hat{\xi}_{i}$. Thus, it is likely that the modal coherency will not be affected if the optimization is carried out for a single variable rather than for two. However, for the purposes of this study both $\hat{\xi}_{i}$ and $\widehat{\omega}_{n, i}$ were optimized.

\subsection{Input Force Estimation}

The modal input estimates for the coherency calculations are obtained from estimates of the time varying tyre forces of the truck as it moves over the bridge. A variety of methods might be used to estimate these spatio-temporal forces which arise out of the interaction of the truck and bridge. For this study, the three methods shown in Figure 5 and described briefly below are primarily used for comparison of simulation results. For the purposes of obtaining force estimates from data gathered in field experiments, Kalman filter estimation, the most accurate of the three methods compared herein, is employed. Details of the methods have been published in a previous study by the authors and may be found in [24]. 


\subsubsection{METHOD A: Static Force estimation}

For this method, the forces acting on the bridge, are assumed to be the static tyre loads of the truck when it is at rest. No dynamic components are added, and the deflection of the bridge has no effect on the tyre load.

\subsubsection{METHOD B: Quarter-Car estimation}

The forces at each wheel are estimated individually by using wheel accelerations obtained either from simulation or measurement. The wheel-assembly is assumed to be a simple mass with the suspension and tyre represented by linear springs. This method captures some dynamics but fails to capture complex spatial interactions between forces at different tyres [24].

\subsubsection{METHOD C:Kalman-Filter estimation}

The tyres as well as the sprung masses are represented by using a pitch-plane model of the truck with 3 lumped axles and 7 degrees of freedom (DoF). The tyres at each axle are assumed to contribute equally to the axle force. Sprung mass linear and pitch velocities, tyre and suspension deflection and axle velocities are used as the states of the Kalman filter. The sensor measurements such as chassis or axle accelerations and suspension deflections which might be measured in the field or obtained from simulation are expressed as function of these states. The Kalman filter procedure is then applied to the state space model to yield tyre forces which can also be expressed as a function of the states. This method has been shown to capture the spatial dynamics better and yield more accurate tyre force estimates in simulation [24].

\subsection{Dealing with spatial dependency of inputs and outputs}

As defined, $\gamma_{m, i}{ }^{2}$ is calculated in the time-domain and defines coherency between input and 
output of a particular mode of a structure. However, the interaction between a vehicle and a structure is more complex than a simple input output relationship. Inputs from the wheels are received by the bridge at multiple points which vary with time as the vehicle moves across the bridge and the response of the bridge as a continuous structure cannot be defined as the response of a single point. To process the temporal and spatial dependencies of the inputs and response and obtain their modal components, we use the procedure illustrated in Figure 6(a).

In this study, we use a reduced order linearized bridge model derived from an original FEM model of a highway bridge over I-275N in Michigan, USA [6]. The FEM model uses the nodal points shown as small red dots in Figure 6(b). The linearized bridge model has fewer degrees of freedom than the FEM model which are represented by a set of generalized coordinates. A set of baseline tyre forces and sensor values are generated by co-simulation of the truck and bridge models. For this purpose, a high fidelity truck model based on a real instrumented truck was created in the multi-body vehicle dynamics simulation software Trucksim $^{\circledR}[16,24]$. Different methods of force estimation described in section 3.3 are used with the simulated vehicle sensor data to obtain the estimated wheel loads which are then interpolated to the nodes. The noise levels in the form of estimation error associated with each method, serve as a test of the coherency estimation procedure [24].

Vehicle sensor data are also available from field tests of the real truck. The Kalman filter estimation process generates a forcing function based on this field data which serves as a more representative and realistic input to the coherency estimation process. Thus after using a suitable force estimation procedure on either real or simulated vehicle sensor data and performing spatial interpolation, the vector of estimated loading of the bridge $\hat{f}^{c}$ is obtained at the nodes as a function of time [24]. 
The modal estimates of the forces $x_{i}$ are then obtained by using:

$$
x_{i}(t)=\mathrm{V}_{i}^{\mathrm{T}} \mathbf{C}^{\mathrm{T}} \underline{\hat{f}}^{c}
$$

where $\mathrm{V}_{i}$ represents the $i^{\text {th }}$ eigen-mode of the linearized bridge model in the generalized coordinate space. $\mathbf{V}=\left[\mathrm{V}_{1} \mathrm{~V}_{2} \ldots \mathrm{V}_{n}\right]$ forms an orthonormal set such that $\mathbf{V}^{\mathrm{T}} \mathbf{M V}=\mathbf{I}$, $\mathbf{I}$ being the identity matrix and $\mathbf{M}$ the mass matrix of the bridge. Here, $\mathbf{C}$ is a transformation matrix from the generalized co-ordinate space to the nodal space such that the $i^{\text {th }}$ mode-shape may be represented in the nodal space as $\mathrm{CV}_{i}$.

The measured nodal acceleration $\underline{\ddot{w}}(t)$ or displacement $\underline{w}(t)$ response of the bridge are similarly, also functions of both time and space and may be decomposed to modal space using the relation:

$$
y_{i}(t)=\mathrm{V}_{i}^{\mathrm{T}} \mathbf{M C}^{\dagger} \underline{\ddot{w}}(t) \quad \text { or } \quad y_{i}(t)=\mathrm{V}_{i}^{\mathrm{T}} \mathbf{M C}^{\dagger} \underline{w}(t)
$$

where $y_{i}$ is the modal response in mode $i . \mathbf{C}^{\dagger}$ is the pseudo-inverse of $\mathbf{C}$ which maps the nodal responses to the space of generalized co-ordinates.

\subsection{Obtaining modal responses from sensor values}

In simulation it is possible to obtain the measured response of each nodal point on the bridge, thus making the transformation in equation (16) possible. However, in field-testing only a limited number of sensors can be mounted at specific locations on the bridge. In this case, the method of recovery of the modal responses depends on the nature of response being measured (displacement or acceleration) and the number of sensors available. If the number of sensors available equals or exceeds the number of modes of the structure. Then the problem is reduced to an exact or overdetermined one. Such a problem is simple to solve algebraically on account of there being more than enough information to determine the modal contributions uniquely. In fact, equation 
(16) might be considered such a solution, if each nodal point is considered to be a sensor.

In this study, we concentrate on the more likely scenario wherein there are fewer sensors than modes. In this case, it is necessary to recover modal responses from sensors measuring displacement, while making further assumptions about the participating modes. Figure 7, shows the RMS signal strength of the response in each mode of linearized bridge model when excited by wheel loads estimated from data obtained from actual runs of the truck on the bridge.

As seen in the Figure 7, only a few global modes with low natural frequencies dominate the displacement response of the structure, as opposed to the acceleration response which possesses significant contributions from higher frequency modes as well. Hence, the problem of extracting modal responses using displacement data is far more tractable than one using acceleration sensor data. If $k$ sensors record the displacement at certain locations on the bridge, the nodal displacement $\underline{w}(t)$ might be recovered as a linear combination of the $p$ dominant modeshapes $\mathbf{C V}_{1}, \mathbf{C V}_{2}, \ldots, \mathbf{C V}_{p}(p \leq k)$ :

$$
\underline{w}(t)=\mathrm{a}_{1}(t) \mathrm{CV}_{1}+\mathrm{a}_{2}(t) \mathrm{CV}_{2}+\cdots+\mathrm{a}_{p}(t) \mathrm{CV}_{p}
$$

wherein the coefficients $\mathrm{a}_{i}(t) ; i=1,2, \ldots p$ might be obtained by a least squares fit of the measured displacement sensor values $\mathrm{s}(t)$ as follows:

$$
\left[\mathrm{a}_{1}(t) \mathrm{a}_{2}(t) \ldots \ldots \mathrm{a}_{p}(t)\right]^{\mathrm{T}}=\left(\mathbf{V}_{s}^{\mathrm{T}}\right)^{\dagger} \mathrm{s}(t)
$$

where the $i-j$ th element of the matrix $\mathbf{V}_{\boldsymbol{s}}$ represents the value of the $i^{\text {th }}$ mode-shape $\left(\mathbf{C} \mathbf{V}_{i}\right)$ at the location of the $j^{\text {th }}$ sensor. The modal displacements may then be obtained from $\underline{w}(t)$ as per equation (16). In practice, the accuracy of the $\mathrm{a}_{i}$ 's obtained might be increased by first filtering $\mathrm{s}(t)$ to remove signal content that is much higher than the expected natural frequency of the $p^{\text {th }}$ mode. 
Although displacement is preferable for extracting the modal responses, it is acceleration data which are usually available, as they are far easier and more economical to measure in the field. It is possible to reconstruct sensor displacements $\mathbf{s}(t)$ directly from accelerations. In simulation, in the absence of any noise, this is achieved by simple double integration. However, in practice this amplifies noise at low frequencies and might cause drift, so filtration of the signals at these frequencies is necessary. This is a well-studied procedure $[25,26]$ and can be carried out by the use of standard techniques such as the FIR filter in [25].

The displacement reconstruction technique in [25] does not estimate the pseudo-static displacements at extremely low frequencies. However, these low frequencies do not significantly affect the transient input-output relationship, which we aim to capture with modal coherency. Hence, to obtain a cleaner result for coherency, in addition to FIR filtering, we completely remove signal content at low frequencies (below $\sim 1 \mathrm{~Hz}$ ) from both the input and output.

\subsection{Pooled coherency}

After, the inputs and outputs at different modes have been obtained it becomes possible to estimate a transfer function $\widehat{H}_{i}$ across each mode to generate the best fit estimates for modal responses $\hat{y}_{i}$ and define modal coherency $\gamma_{m, i}{ }^{2}$. In addition, it is possible to capture the coherency across a set of modes. A pooled squared coherency may be defined as:

$$
\gamma_{p}^{2}:=\sum_{i=1}^{m} q_{i} \gamma_{m, i}^{2}
$$

where $\quad q_{i}=\left|\bar{Y}_{i}\right| /\left(\sum_{i=1}^{m}\left|\bar{Y}_{i}\right|\right)$ and $\quad \bar{Y}_{i}=\left(\sum_{t=0}^{t_{f}} y_{i}^{2}(t)\right) / N_{t}$

Thus $\gamma_{p}{ }^{2}$ is the weighted sum of the modal coherencies, where the weights are the elements of the $\mathrm{L}^{1}$ normalized vector of mean squared values of the measured outputs of the 
corresponding modes $1,2 \ldots, m$. Because $\gamma_{m, i}{ }^{2}$ lie between 0 and 1 , and the weights are normalized, it follows that $\gamma_{p}{ }^{2}$ also lies between 0 and 1. By this definition, $\gamma_{p}{ }^{2}$ amplifies the coherencies of the modes contributing most significantly to the response and tends towards 1 when they show high modal coherency.

\section{RESULTS}

\subsection{Simulation Results assuming knowledge of nodal accelerations}

The performance of three force estimators, Methods (A), (B) and(C) discussed in section 3.3, when applied to the sensor information obtained from simulation in Trucksim $^{\circledR}$, is compared in Figure 8. It is known that $(\mathrm{C})$ is a better estimator of the dynamic forces than $(\mathrm{B})$, while estimation by (A) is even poorer [24]. As an example, the figure shows the response of the linearized bridge model to forces and estimated forces for a single mode. To obtain these, knowledge of nodal values of responses is assumed. The estimated 'true' response of the mode is known from simulation (shown in blue). The behavior is roughly similar in other modes as well.

Table 1 gives the values of $\gamma_{m, i}{ }^{2}$ using the three estimation procedures in each of the modes. It is seen that as more accurate estimation procedures are used, the responses in each mode become more accurate. As a consequence, the pooled coherency values increase as well (see Figure 8 caption).

\subsection{Results from realistic force estimates and simulated sensor accelerations}

Figures 9 and 10 show the performance of the Kalman filter estimation procedure for data collected from the field test of an instrumented truck on the actual bridge which was modeled. Raw sensor data such vertical acceleration of the truck's tractor while it travels over the bridge 
shown in Figure 9(a) is used as an input to the filter. The Kalman filter employs the pitch plane model in addition to the sensor data to compute the evolution of its internal states such as the rear trailer axle deflections shown in Figure 9(b).A linear combination of these states yields the desired axle force estimates shown in Figure 9(c). The states can also be combined to yield quantities that might be used for validation against experimentally measured values such as the $5^{\text {th }}$ wheel (hitch) vertical loads and tractor pitch rates shown in Figure 10.

Although, in this situation the measured quantities serve as a good tool for comparison with the estimated ones, there is no 'ground truth' of actual tyre forces available for verification. Since the states contributing to the $5^{\text {th }}$ wheel forces and the tractor pitch rates in the Kalman filter are different from those which contribute to the tyre forces, their match does not conclusively establish the accuracy of tyre load estimates. It is in such a situation, that coherency helps us verify the force estimation procedure. Since reliable results for sensor values from the bridge are not available, we assume outputs of the linearized bridge model excited by these force estimates, to demonstrate the procedure. Sensor accelerations from the locations shown as numbered large green dots in Figure 6(b) are assumed and $\gamma_{m, i}{ }^{2}$ and $\gamma_{p}{ }^{2}$ are estimated between the estimated forces and reconstructed displacements.

As shown in Figure 11, $\gamma_{m, 4}{ }^{2}$ is 0.981 in mode 4 which dominates the displacement response due to the filtered input forces. Although, the $\gamma_{m, i}{ }^{2}$ value is lower for other modes, the contributions of these modes to the net dynamic displacement are comparatively much lower. Hence, the overall coherence does not drop much, as evidenced by the value of $\gamma_{p}{ }^{2}$ which for this case is 0.973 .

Table 2 shows how the estimation of $\gamma_{p}{ }^{2}$ is affected as different levels of random noise are added to the simulated acceleration sensor signals. Since the measures are time-based 
estimates, the same Gaussian pseudo-random time-series with different amplitudes was used as noise in each case. As expected, we see a monotonic decrease in coherency with output noise.

\section{CONCLUSIONS}

In order to address specific demands of vehicle-structure interaction, new measures of modal and pooled squared coherency, $\gamma_{m, i}{ }^{2}$ and $\gamma_{p}{ }^{2}$, have been defined. These measures were motivated as a means of validating the accuracy of tyre load estimates in the absence of ground-truth knowledge of 'true' tyre loads. To calculate these coherency measures, tire loads should be estimated on the truck and measurements of bridge response at a set of sensor locations need to be made. In the above, both simulated and experimental tyre load estimates have been used. However, for simplicity in this work, simulated bridge deflection have been used throughout; the emphasis here has been on methodology and developing analysis tools; a full experimental study is deferred to future work.

The procedure for coherency estimation requires some prior knowledge about the characteristics of the structure, i.e. the dominant mode-shapes and approximate natural frequencies. Here it has been shown by extensive simulation, and using realistic forcing inputs, that the coherency measures are well suited to handle many of the issues faced during field tests, such as short measurement time, limited sensor information and low structural damping. The sensitivity to both input and output noise has also been detailed in this paper. Overall, these measures provide new tools for validation of estimation and measurement procedures involving interactions between dynamic loads and flexible structures. In the future, simultaneously collected bridge and vehicle data may be used to validate the procedure for different bridge structures and vehicle types. Certain theoretical aspects of the problem are also subject to further study, such as the sensitivity to sensor placement and to the details of the optimization routine. 


\section{Acknowledgements}

The authors are extremely grateful to Dr. Yun Hwa Hong for his help and explanations regarding displacement reconstruction using FIR filters. We also gratefully acknowledge the generous support offered by the U.S. Department of Commerce, National Institute of Standards and Technology (NIST) Technology Innovation Program (TIP) and the assistance of Michigan Department of Transportation for providing access to bridge and for collecting data.

\section{References}

[1] Sohn H., Farrar C.R., Hemez F.M., Shunk D.D.,et.al., “A review of structural health monitoring literature: 1996-2001”, Los Alamos National Laboratory report LA-13976MS, 2004.

[2] Senthilvasan, J., Thambriatnam, D. P. and Brameld, G. H., "Dynamic response of a curved bridge under moving truck load," Engineering Structures 24(10), 1283-1293 (2002)

[3] Harris N. K., Obrien E. J. and Gonalez A., "Reduction of bridge dynamic amplification through adjustment of vehicle suspension damping." Journal of Sound and Vibration 302 (2007), 471-485.

[4] Wu S. Q. and Law S. S., "Evaluating the response statistics of an uncertain bridge-vehicle system”, Mechanical Systems and Signal Processing, 27 (2012), 576-589.

[5] Tuononen., A.J., "On-board estimation of dynamic tyre forces from optically measured tyre carcass deflections," International Journal of Heavy Vehicle Systems 2009, volume 16, number 3, p.p. 362-378.

[6] Tuononen., A.J., “Optical position detection to measure tyre carcass deflections" Vehicle System Dynamics 2008, volume 46, number 6, p.p. 471-481

[7] Ray, L.R., "Nonlinear state and tire force estimation for advanced vehicle control," Control Systems Technology, IEEE Transactions on , vol.3, no.1, pp.117-124, Mar 1995, doi:10.1109/87.370717 
[8] Khemoudj, O., Imine, H., and Djemai M., "Variable gain sliding mode observer for heavy duty vehicle tyre forces estimation," (paper presented in 11th International Workshop on Variable Structure Systems, VSS'10 Mexico city ,Mexico June 26-28, 2010)

[9] Khemoudj, O. , Imine, H., and Djemai, M., "Heavy duty vehicle tire forces estimation using variable gain sliding mode observer," International Journal of Vehicle Design, special issue "Variable Structure System in automotive application",2012

[10] Imine, H., Djemai, M., Khemoudj, O., and Germanchev, A., "Sliding mode observers for tyre vertical forces estimation: experimental results using ALF," (paper presented in International Symposium on Heavy Vehicle Transport Technology, Stockholm, Sweden September 16-19, 2012)

[11] Deng., L., and Cai, C.S., "Identification of Dynamic Vehicular Axle Loads: Theory and Simulations", Journal of Vibration and Control, December, 2010 16: 2167-2194, first published on May 26, 2010, doi:10.1177/1077546309351221

[12] Huang D.Z., Wang T.L., "Vibration of highway steel bridge with longitudinal grades." Computers and Structures, 69 (1998), pp. 235

[13] Zhu, X.Q., and Law, S.S., "Identification of vehicle axle loads from bridge dynamic responses" Journal of Sound and Vibration, Volume 236, Issue 4, 28 September 2000, p.p 705-724, doi: 10.1006/jsvi.2000.3021

[14] Pinkaew., T., "Identification of vehicle axle loads from bridge responses using updated static component technique," Engineering Structures, Volume 28, Issue 11, September 2006, p.p. 1599-1608, doi: 10.1016/j.engstruct.2006.02.012.

[15] Zhu, X.Q., and Law, S.S., "Dynamic axle and wheel loads identification: laboratory studies" Journal of Sound and Vibration, Volume 268, Issue 5, 11 December 2003, p.p 855-879, doi: 10.1016/S0022-460X(02)01557-2

[16] Mosavi A.A., Mitra M., van der Linden G.W, Gordon T., et al., "Nonlinear modeling of the vehicle/structure interaction on a skewed highway bridge using an iterative uncoupled approach,” Proceedings of SPIE Vol. 8347, 834728 (2012), doi: 10.1117/12.916187

[17] Tan G.H.; Brameld G.H.; Thambiratnam D.P., "Development of an analytical model for treating bridge-vehicle interaction," Engineering Structures, Volume 20, Number 1, January 1998 , pp. 54-61(8) 
[18] Kalman, R.E., "A new approach to linear filtering and prediction problems", Trasactions of the ASME: Journal of Basic Engineering, vol 82, Series D, pp. 35-45, 1960.

[19] Sun Y.Q., Dhanasekar M., "A dynamic model for the vertical interaction of the rail track and wagon system.” International J. Solids Struct., 39: 1337-1359 (2002)

[20] Yang Y. B., Yau J. D. and Wu Y. S., Vehicle Bridge Interaction Dynamics with application to high speed railways, World Scientific Publishing Co. Pte. Ltd. 2004

[21] Bendat., J.S., Piersol., A.G., Random Data: Analysis and Meaurement Procedures, , Fourth Edition, John Wiley \& Sons, Inc., Hoboken, NJ, USA. ,1986, p.p. 164-252

[22] Welch, P., "The use of fast fourier transform for the estimation of power spectra: A method based on time averaging over short, modified periodograms," Audio and Electroacoustics, IEEE Transactions on, vol.15, no.2, pp. 70- 73, June 1967,doi: 10.1109/TAU.1967.1161901

[23] Gubner., J.A., Probability and Random Processes for Electrical and Computer Engineers, Cambridge University Press, 2006, p.p. 92

[24] Gordon, T. and Mitra, M., "Dynamic Load Estimation for Heavy Trucks on Bridge Structures,” SAE Int. J. Commer. Veh. 6(1):62-72, 2013, doi:10.4271/2013-01-0626

[25] Hong Y.H, Kim H., Lee H.S, "Reconstruction of dynamic displacement and velocity from measured accelerations using the variational statement of an inverse problem", Journal of Sound and Vibration, 329 (2010) pp. 4980-5003, 2010.

[26] Lee H.S, Hong Y.H and Park H.W, "Design of an FIR filter for the displacement reconstruction using measured acceleration in low-frequency dominant structures," International Journal for Numerical Methods in Engineering, Vol. 82, No. 4, 403-434, 2010 
Table 1 Modal Squared Coherency $\left(\gamma_{m, i}{ }^{2}\right)$ values for three force estimation methods for different modes

\begin{tabular}{|c|c|c|c|c|c|c|c|c|c|c|}
\hline $\begin{array}{c}\text { Mode no } \\
(i)\end{array}$ & 1 & 2 & 3 & 4 & 5 & 6 & 7 & 8 & 9 & 10 \\
\hline A & 0.994 & 0.962 & 0.917 & 0.190 & 0.087 & 0.150 & 0.188 & 0.188 & 0.104 & 0.194 \\
\hline B & 0.987 & 0.898 & 0.971 & 0.754 & 0.074 & 0.881 & 0.754 & 0.437 & 0.211 & 0.039 \\
\hline C & 0.999 & 0.981 & 0.999 & 0.989 & 0.256 & 0.998 & 0.969 & 0.740 & 0.969 & 0.842 \\
\hline
\end{tabular}

17 
Table 2 Pooled Squared Coherency $\left(\gamma_{p}{ }^{2}\right)$ values for different levels of random noise added to sensor accelerations

\begin{tabular}{|c|c|c|c|c|c|c|}
\hline Noise to signal ratio & $0 \%$ & $2 \%$ & $4 \%$ & $6 \%$ & $8 \%$ & $10 \%$ \\
\hline$\gamma_{p}{ }^{2}$ & 0.973 & 0.954 & 0.888 & 0.748 & 0.520 & 0.491 \\
\hline
\end{tabular}




\section{List of Figure Captions}

Figure 1(a) Classical squared coherency estimate with FFT window length $=0.5 \mathrm{~s}$ and high damping ratio $(\zeta=0.7$, settling time $=0.09 \mathrm{~s})$

Figure 1(b) Classical squared coherency estimate with FFT window length $=0.5 \mathrm{~s}$ and low damping ratio $(\zeta=0.07$, settling time $=0.91 \mathrm{~s})$

Figure 2 Estimation of modal squared coherency

Figure 3(a) Variation of Modal squared coherency with Input noise to signal ratio

Figure 3(b) Variation of Modal squared coherency Output noise to signal ratio

Figure 4(a) Objective function surface for mode 4 without input or output noise (minima at red dot at damping ratio 0.0007 and natural frequency $14.1438 \mathrm{rad} / \mathrm{s}$ )

Figure 4(b) Objective function surface for mode 4 with random input and output noise at 5\% noise to signal ratio (minima at red dot at damping ratio 0.0007 and natural frequency 14.1438 $\mathrm{rad} / \mathrm{s})$

Figure 5 Force Estimation Methods

Figure 6(a) Modal analysis and decomposition of forcing inputs

Figure 6(b) FEM nodal points and physical sensor locations

Figure 7(a) RMS modal components of displacement (modes arranged from left to right in order of increasing natural frequency)

Figure 7(b) RMS modal components of acceleration (modes arranged from left to right in order of increasing natural frequency)

Figure 8(a) Mode 6 acceleration time-response estimates compared with the actual response for Method A: Static load estimate (Pooled squared coherency across 10 modes $=0.024$ ) 
Figure 8(b) Mode 6 acceleration time-response estimates compared with the actual response for Method B: Quarter-car estimate (Pooled squared coherency across 10 modes $=0.706$ )

Figure 8(c) Mode 6 acceleration time-response estimates compared with the actual response for Method C: Kalman filter estimate (Pooled squared coherency across 10 modes $=0.986$ )

Figure 9(a) Measured vertical acceleration at tractor chassis

Figure 9(b) Estimated suspension deflection of rear trailer axle

Figure 9(c) Estimated rear trailer axle vertical force

Figure 10(a) Estimated and Measured $5^{\text {th }}$ wheel (hitch) forces for field test of truck on bridge

Figure 10(b) Estimated and Measured Tractor pitch rates for field test of truck on bridge

Figure 11(a) Modal displacement time-responses obtained from sensor accelerations for Mode 4 (Modal squared coherency $=0.981$ )

Figure 11(b) Modal displacement time-responses obtained from sensor accelerations for Mode 6 (Modal squared coherency $=0.580$ ) 
1
2
3
4

5

6

7

8

9

10

11

12

13

14

15

16

17

18

19

20

21

22

23

24

25

26

27

28

29

30

31

32

33

34

35

36

37

38

39

40

41

42

43

44

45

46

47

48

49

50

51

52

53

54

55

56

57

58

59

60

\section{Word Count: 5164}

URL: http://mc.manuscriptcentral.com/nvsd 


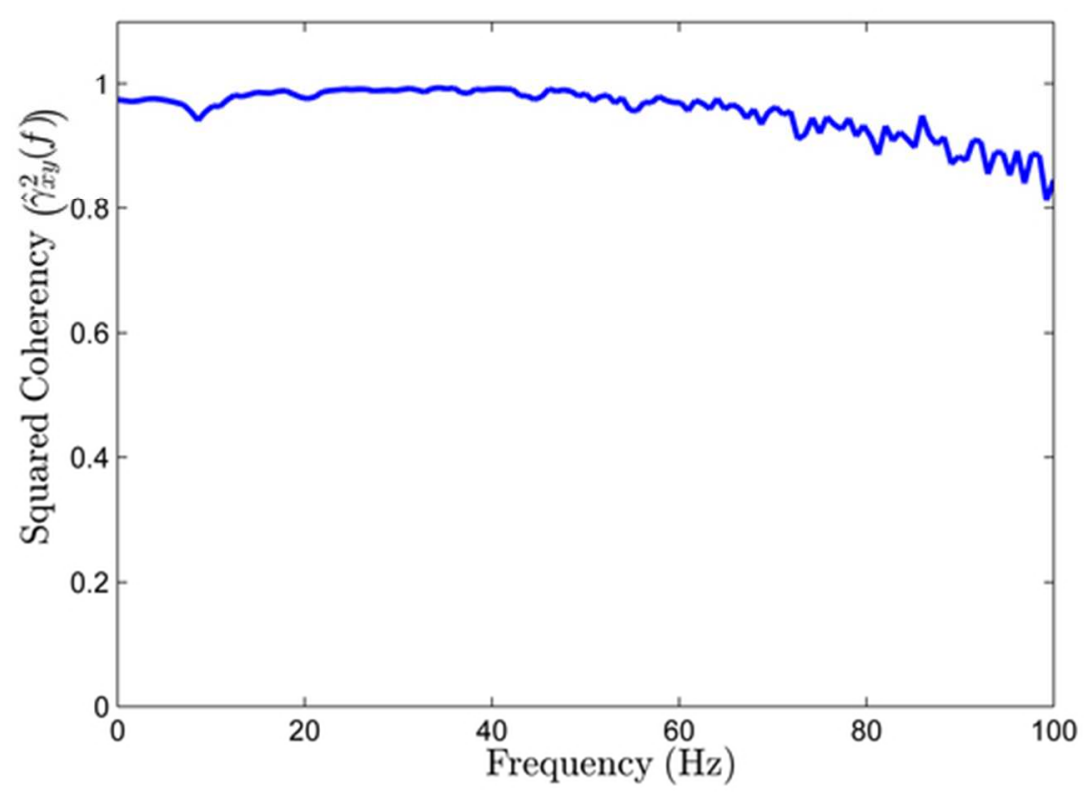
Figure 1(a) Classical squared coherency estimate with FFT window length $=0.5 \mathrm{~s}$ and high damping ratio $(\zeta$ $=0.7$, settling time $=0.09 \mathrm{~s}$ ) $26 \times 17 \mathrm{~mm}(600 \times 600 \mathrm{DPI})$


1

2

3

4

5

6

7

8

9

10

11

12

13

14

15

16

17

18

19

20

21

22

23

24

25

26

27

28

29

30

31

32

33

34

35

36

37

38

39

40

41

42

43

44

45

46

47

48

49

50

51

52

53

54

55

56

57

58

59

60

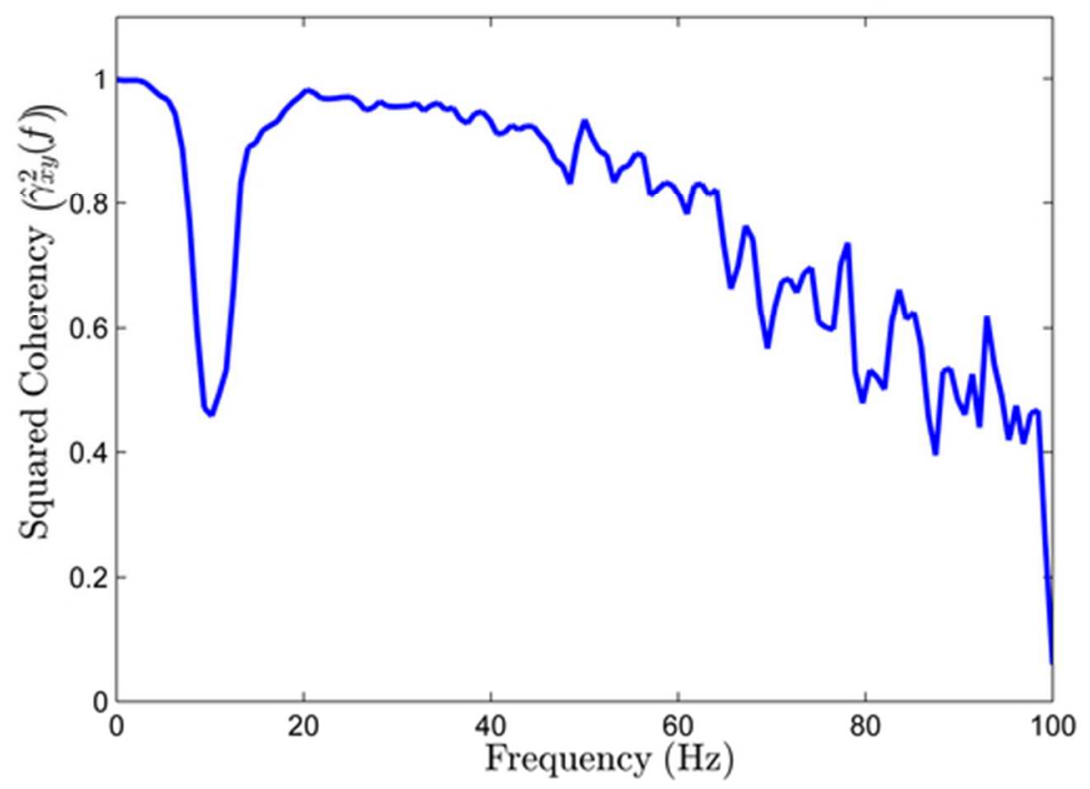

Figure 1(b) Classical squared coherency estimate with FFT window length $=0.5$ s and low damping ratio $(\zeta=$ 0.07 , settling time $=0.91 \mathrm{~s})$ $26 \times 17 \mathrm{~mm}(600 \times 600 \mathrm{DPI})$ 
Figure 2 Estimation of modal squared coherency $38 \times 28 \mathrm{~mm}(600 \times 600 \mathrm{DPI})$ 
1

2

3

4

5

6

7

8

9

10

11

12

13

14

15

16

17

18

19

20

21

22

23

24

25

26

27

28

29

30

31

32

33

34

35

36

37

38

39

40

41

42

43

44

45

46

47

48

49

50

51

52

53

54

55

56

57

58

59

60

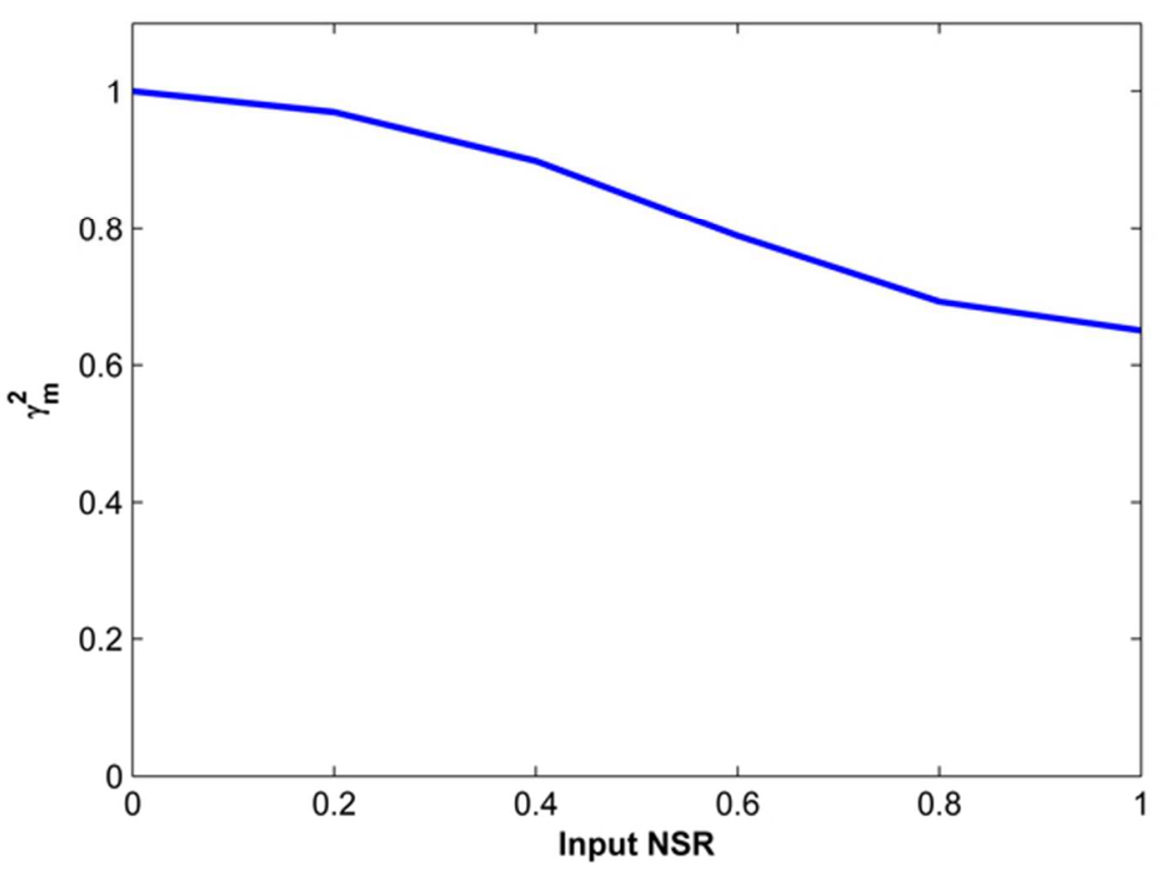

Figure 3(a) Variation of Modal squared coherency with Input noise to signal ratio $28 \times 20 \mathrm{~mm}(600 \times 600 \mathrm{DPI})$ 


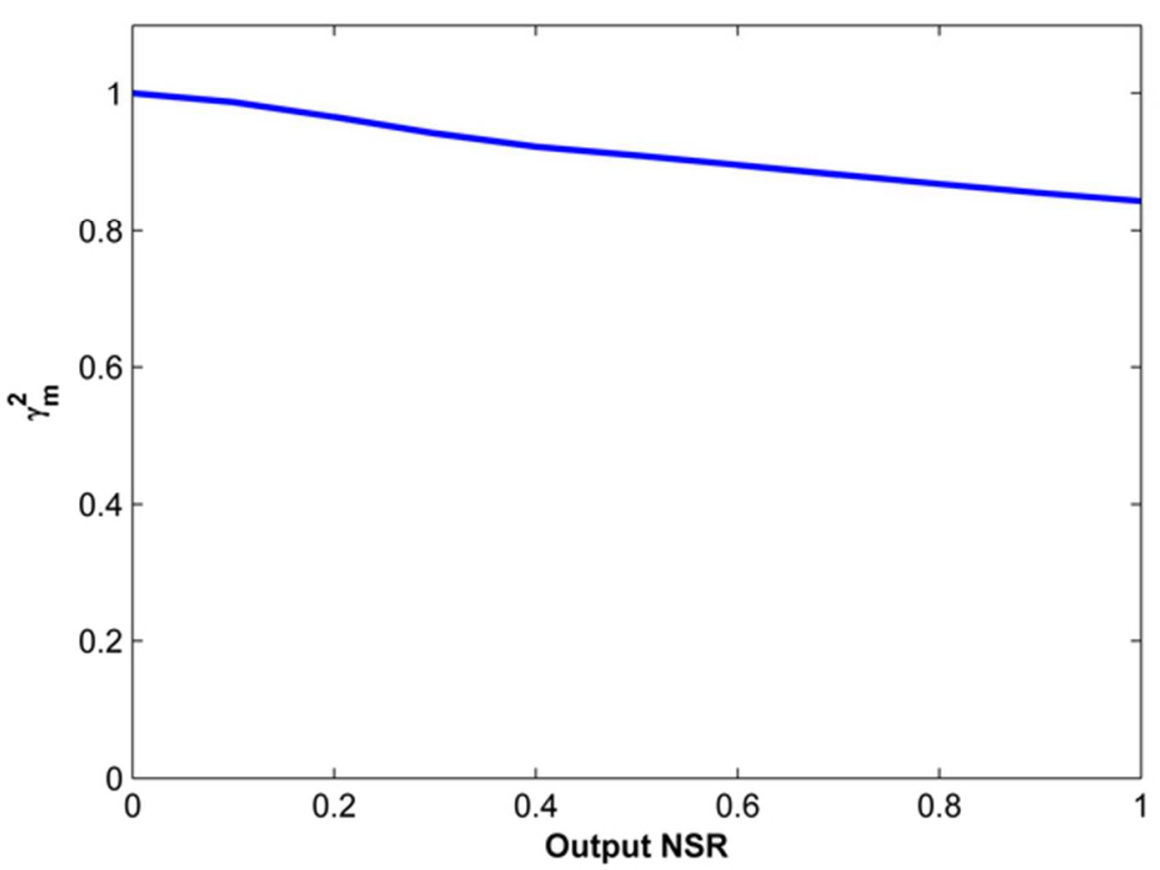

Figure 3(b) Variation of Modal squared coherency Output noise to signal ratio $28 \times 20 \mathrm{~mm}(600 \times 600 \mathrm{DPI})$ 
Figure 4(a) Objective function surface for mode 4 without input or output noise (minima at red dot at damping ratio 0.0007 and natural frequency $14.1438 \mathrm{rad} / \mathrm{s}$ ) $26 \times 17 \mathrm{~mm}(600 \times 600 \mathrm{DPI})$ 
Figure 4(b) Objective function surface for mode 4 with random input and output noise at $5 \%$ noise to signal ratio (minima at red dot at damping ratio 0.0007 and natural frequency $14.1438 \mathrm{rad} / \mathrm{s}$ ) $26 \times 17 \mathrm{~mm}(600 \times 600 \mathrm{DPI})$ 

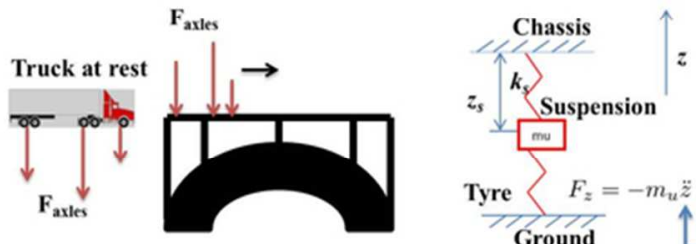

A Static Load Estimate

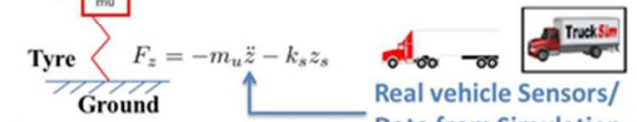

B Quarter-car Estimate (Dehicle Sensors/

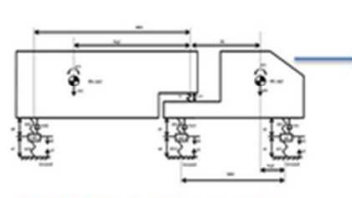

7 DoF Pitch Plane model

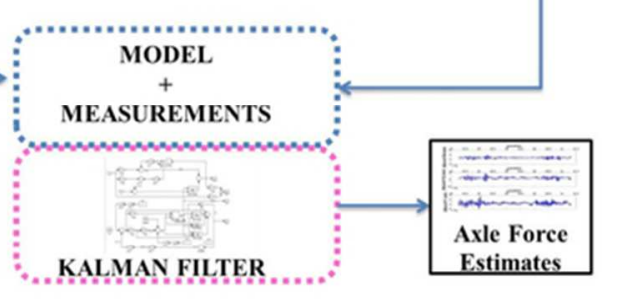

Kalman Filter Estimate

Figure 5 Force Estimation Methods $30 \times 22 \mathrm{~mm}(600 \times 600$ DPI $)$ 
Figure 6(a) Modal analysis and decomposition of forcing inputs $30 \times 22 \mathrm{~mm}(600 \times 600 \mathrm{DPI})$ 


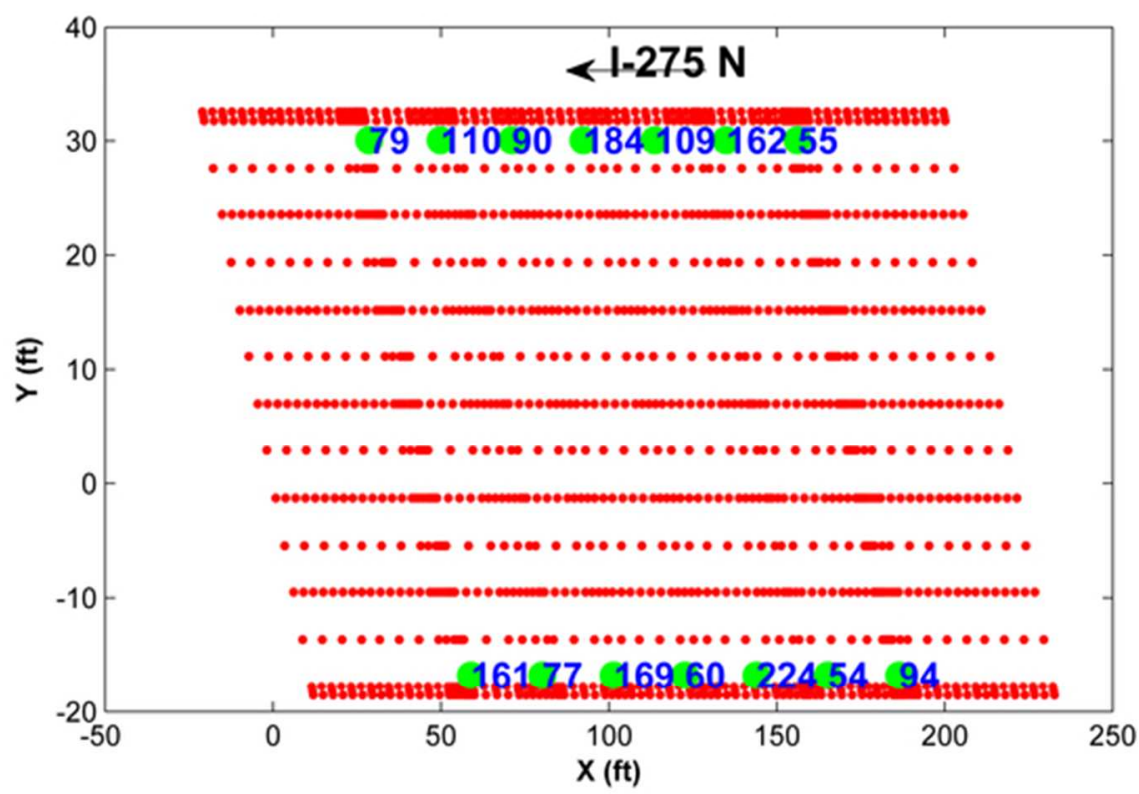

Figure 6(b) FEM nodal points and physical sensor locations $26 \times 17 \mathrm{~mm}(600 \times 600 \mathrm{DPI})$ 


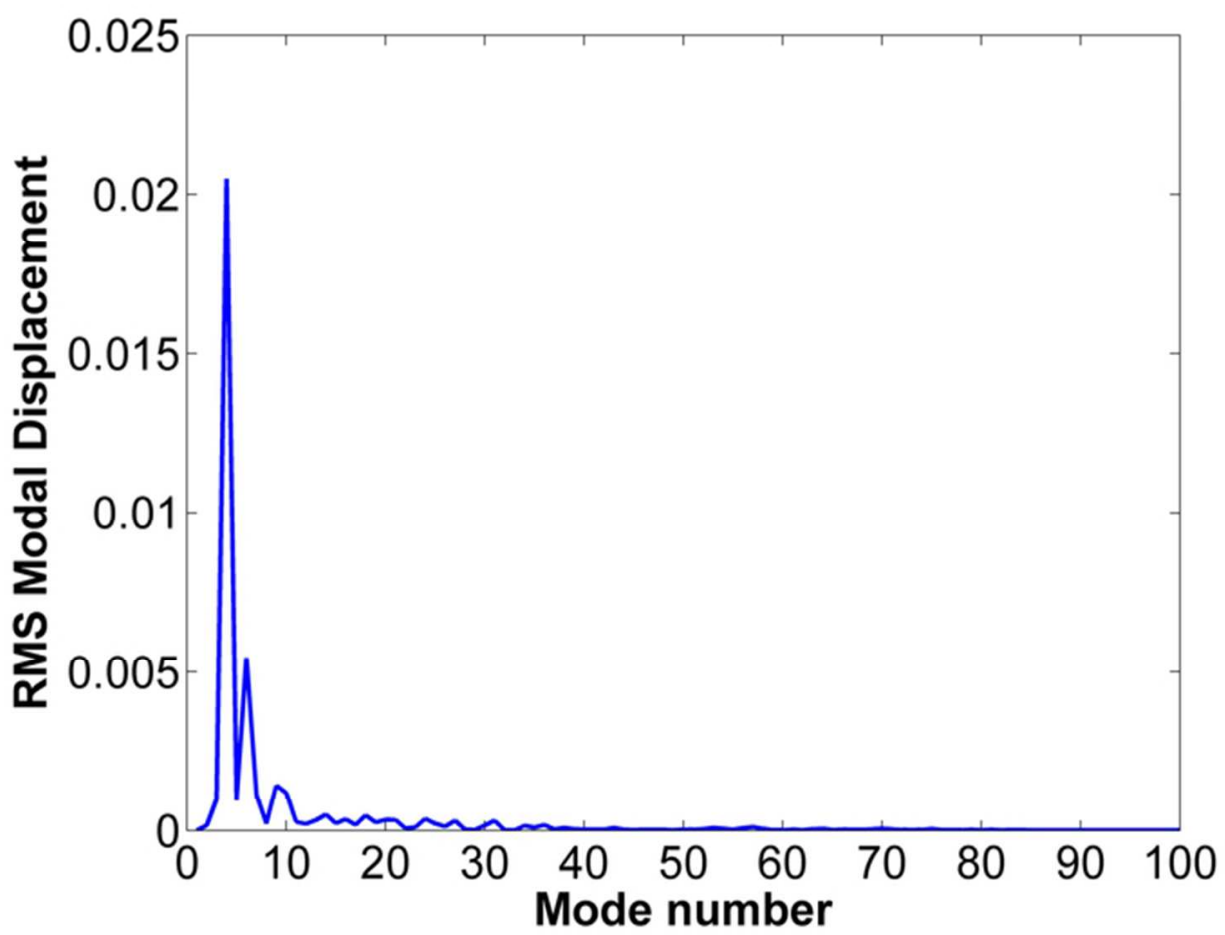

Figure 7(a) RMS modal components of displacement (modes arranged from left to right in order of increasing natural frequency) $30 \times 22 \mathrm{~mm}(600 \times 600 \mathrm{DPI})$ 
1

2

3

4

5

6

7

8

9

10

11

12

13

14

15

16

17

18

19

20

21

22

23

24

25

26

27

28

29

30

31

32

33

34

35

36

37

38

39

40

41

42

43

44

45

46

47

48

49

50

51

52

53

54

55

56

57

58

59

60

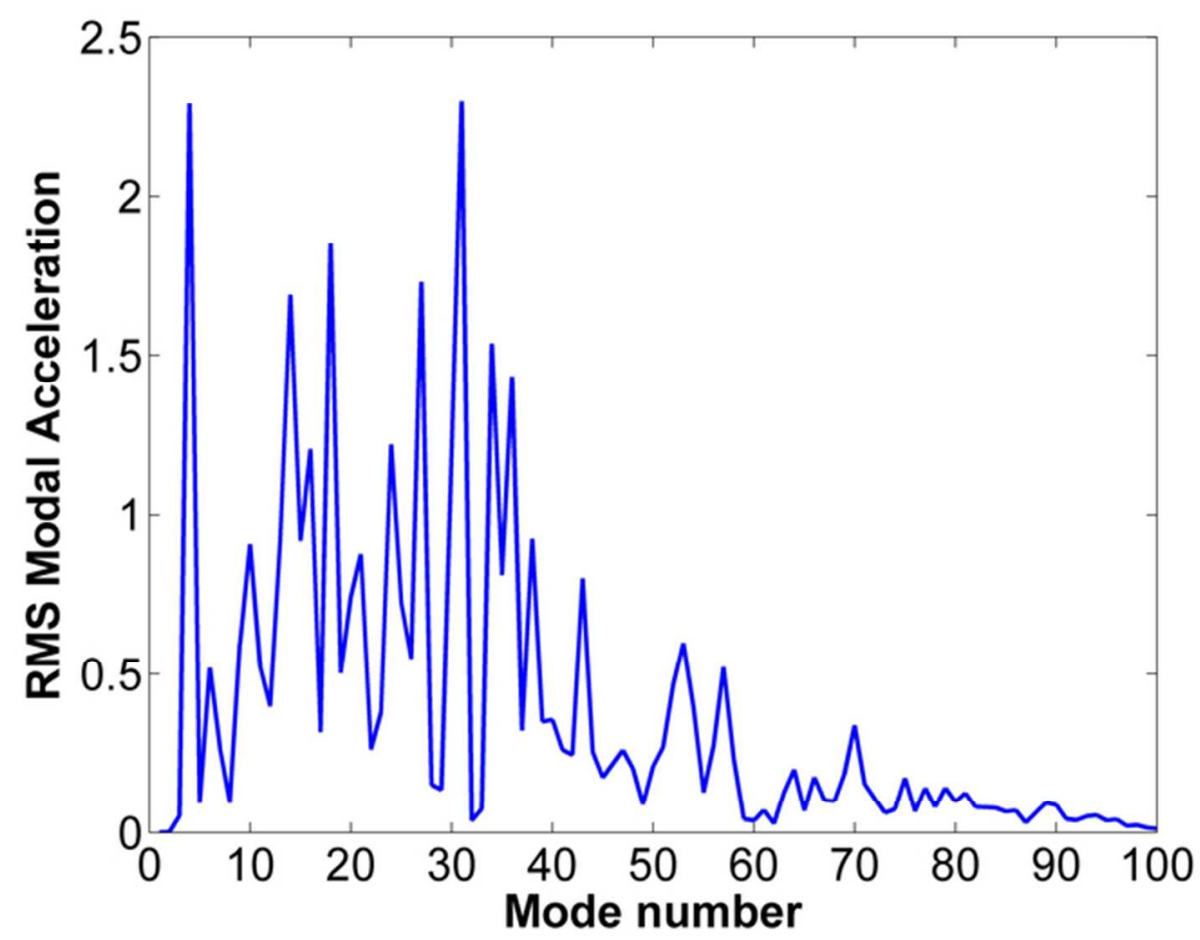

Figure 7(b) RMS modal components of acceleration (modes arranged from left to right in order of increasing natural frequency)

$30 \times 22 \mathrm{~mm}(600 \times 600$ DPI $)$ 


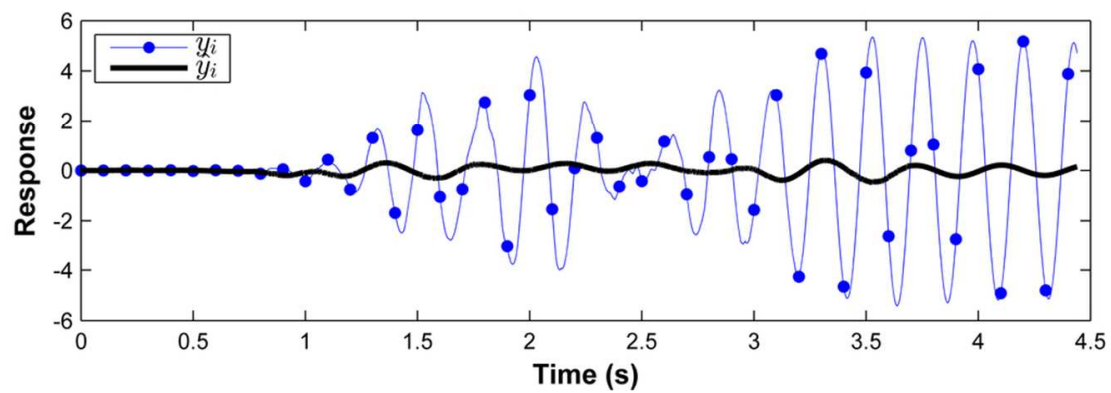

Figure 8(a) Mode 6 acceleration time-response estimates compared with the actual response for Method A: Static load estimate ( Pooled squared coherency across 10 modes $=0.024$ ) $50 \times 15 \mathrm{~mm}(600 \times 600 \mathrm{DPI})$ 
1

2

3

4

5

6

7

8

9

10

11

12

13

14

15

16

17

18

19

20

21

22

23

24

25

26

27

28

29

30

31

32

33

34

35

36

37

38

39

40

41

42

43

44

45

46

47

48

49

50

51

52

53

54

55

56

57

58

59

60

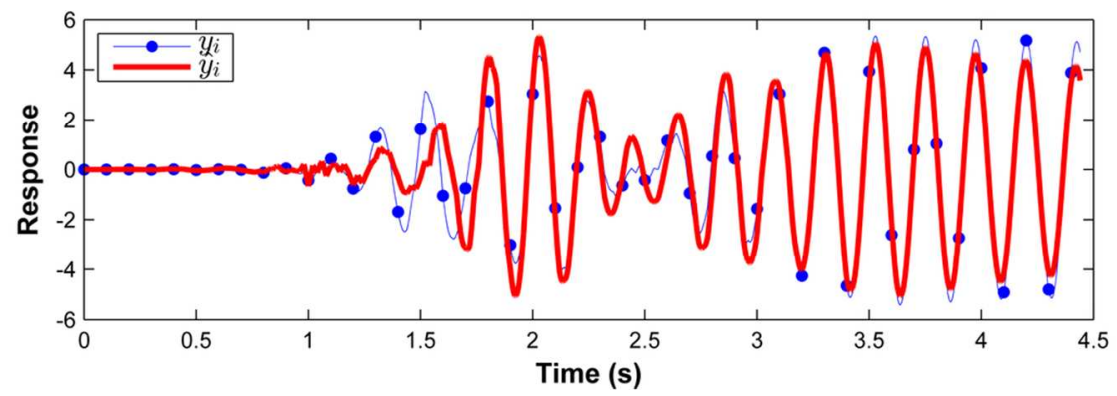

Figure 8(b) Mode 6 acceleration time-response estimates compared with the actual response for Method B: Quarter-car estimate (Pooled squared coherency across 10 modes $=0.706$ ) $50 \times 15 \mathrm{~mm}(600 \times 600 \mathrm{DPI})$ 


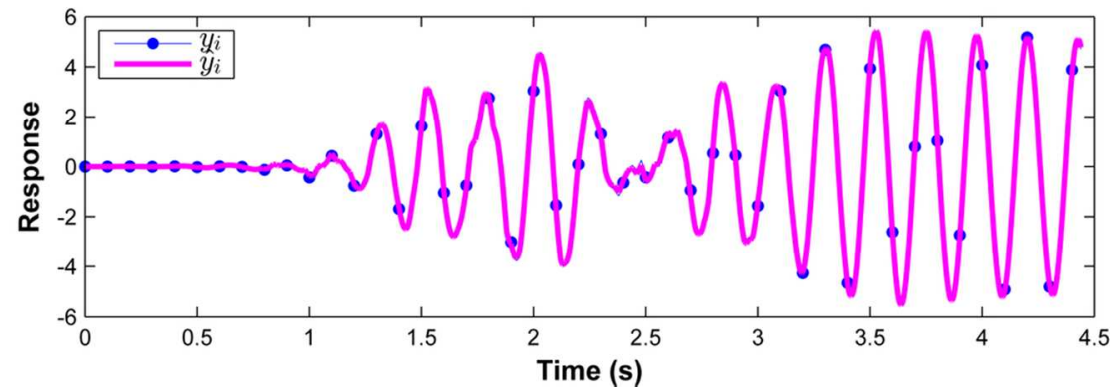

Figure 8(c) Mode 6 acceleration time-response estimates compared with the actual response for Method C: Kalman filter estimate (Pooled squared coherency across 10 modes $=0.986$ ) $50 \times 15 \mathrm{~mm}(600 \times 600 \mathrm{DPI})$ 


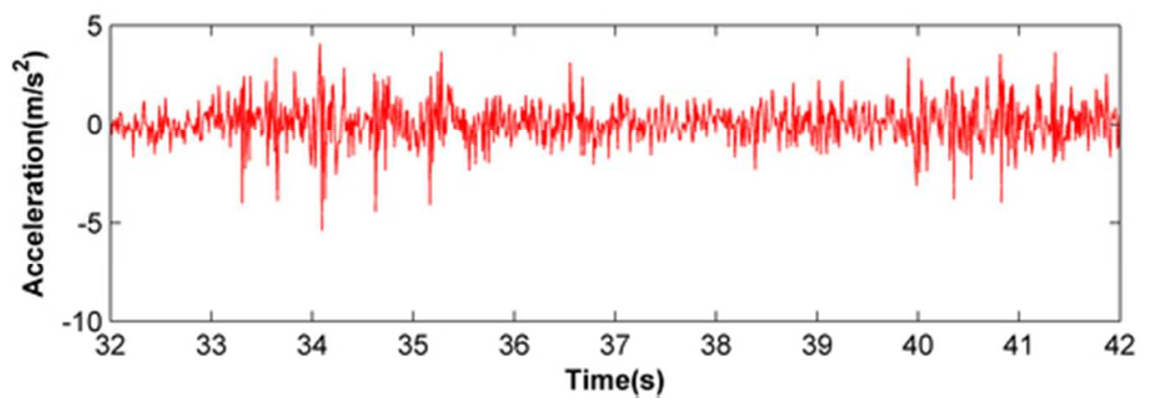

Figure 9(a) Measured vertical acceleration at tractor chassis $50 \times 15 \mathrm{~mm}(300 \times 300 \mathrm{DPI})$ 


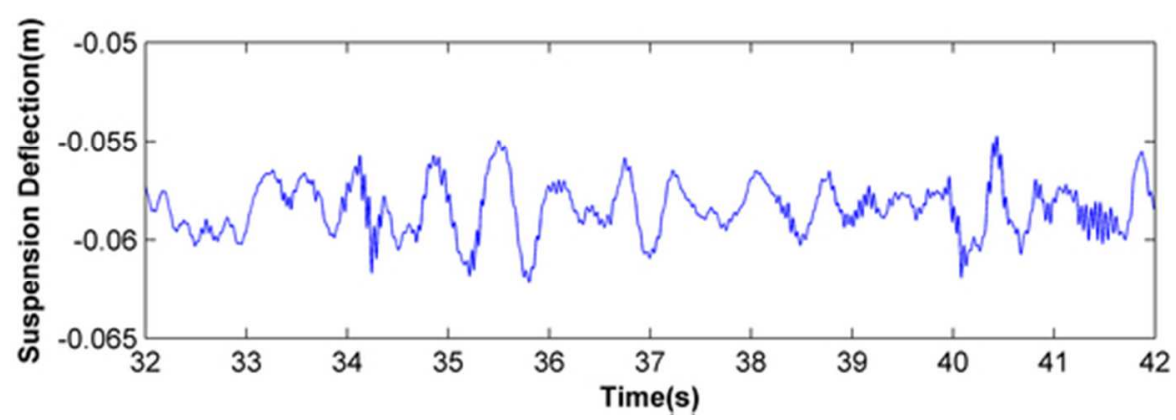

Figure 9(b) Estimated suspension deflection of rear trailer axle $50 \times 15 \mathrm{~mm}(300 \times 300$ DPI $)$ 
1

2

3

4

5

6

7

8

9

10

11

12

13

14

15

16

17

18

19

20

21

22

23

24

25

26

27

28

29

30

31

32

33

34

35

36

37

38

39

40

41

42

43

44

45

46

47

48

49

50

51

52

53

54

55

56

57

58

59

60

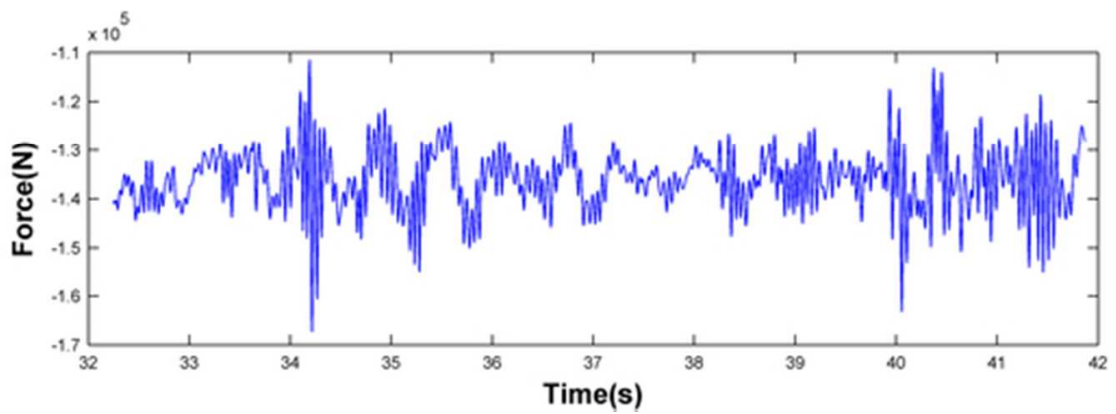

Figure 9 (c) Estimated rear trailer axle vertical force $50 \times 15 \mathrm{~mm}(300 \times 300 \mathrm{DPI})$ 


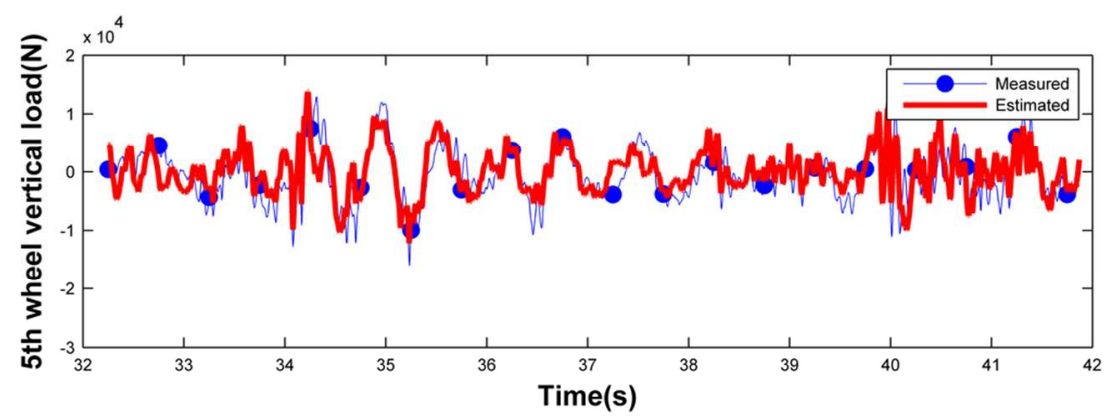

Figure 10(a) Estimated and Measured 5th wheel (hitch) forces for field test of truck on bridge $50 \times 15 \mathrm{~mm}(600 \times 600 \mathrm{DPI})$ 


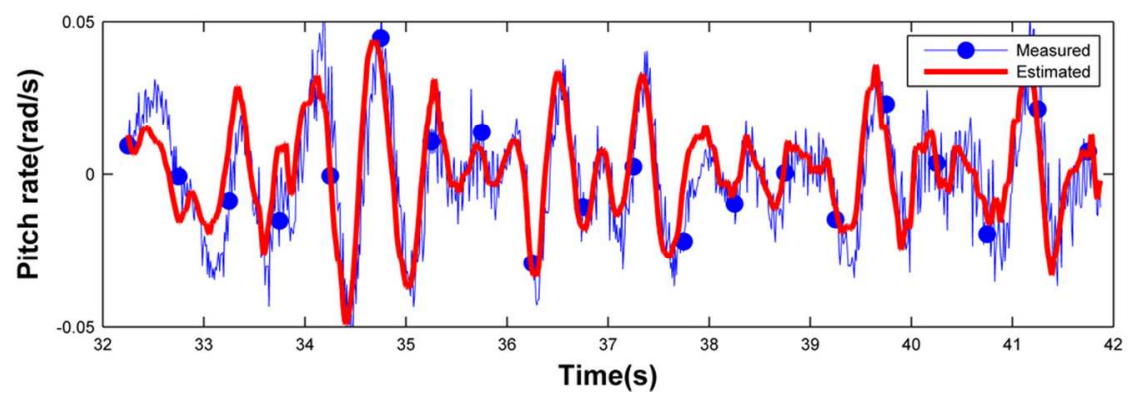

Figure 10(b) Estimated and Measured Tractor pitch rates for field test of truck on bridge $50 \times 15 \mathrm{~mm}(600 \times 600 \mathrm{DPI})$ 


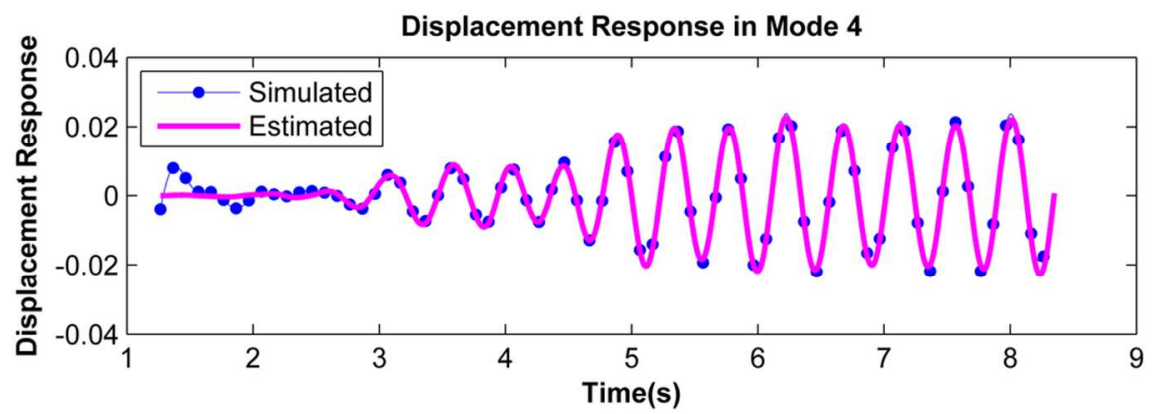

Figure 11(a) Modal displacement time-responses obtained from sensor accelerations for Mode 4 (Modal squared coherency $=0.981)$ $50 \times 15 \mathrm{~mm}(600 \times 600 \mathrm{DPI})$ 
1

2

3

4

5

6

7

8

9

10

11

12

13

14

15

16

17

18

19

20

21

22

23

24

25

26

27

28

29

30

31

32

33

34

35

36

37

38

39

40

41

42

43

44

45

46

47

48

49

50

51

52

53

54

55

56

57

58

59

60

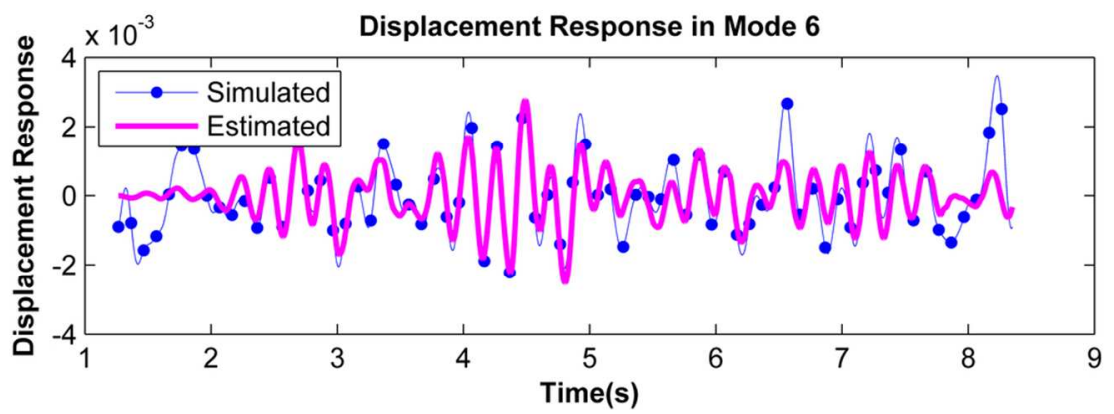

Figure 11(b) Modal displacement time-responses obtained from sensor accelerations for Mode 6 (Modal squared coherency $=0.580$ )

$50 \times 15 \mathrm{~mm}(600 \times 600 \mathrm{DPI})$ 